\title{
Estimação da equação demanda de importação de bens intermediário para o Brasil no período de 1995 a 2008: um modelo de correção de erro
}

Adão Aparecido Ferreira RODRIGUES

Mestre em Economia Aplicada UFRGS. Docente da Universidade Vale do Rio Verde - UninCor, responsável pelas disciplinas: Economia, Microeconomia e Estatística II. E- mail: adaoafr@ hotmail.com

RESUMO: O objetivo desse artigo é estimar a demanda por importação de bens intermediário, com base no método de estimação proposto por Engler e Granger (1987). No período do primeiro trimestre de 1995 ao segundo trimestre de 2008. Os resultados obtidos mostram que as séries são todas integradas de mesma ordem (I (1)), cointegram e então aplicada o mecanismo de correção de erro, obtêm-se elasticidades aceitáveis, mas com divergências em relação a trabalhos anteriores. Que pode ser devido ao período estudado, ter passado por intensas mudanças exógenas.

Palavras - chave: Demanda de importação, Engle e Granger, comércio internacional, raiz unitária.

\section{Estimation of demand equation intermediario imports of goods to Brazil in 1995 to 2008 period: a model for error correction}

\begin{abstract}
The aim of this paper is to estimate the demand for intermediate goods imports, based on the estimation method proposed by Engle and Granger (1987). During the first quarter of 1995 to second quarter 2008. The results show that the series are all integrated of the same order (I (1)), cointegram and then applied the mechanism of error correction, we obtain elasticity's acceptable, but with differences in relation to previous work. That may be due to the study period, have passed through intense exogenous changes.
\end{abstract}

Key - words: Demand for imports, Engle and Granger, international trade, unit root.

\section{INTRODUÇÃO}

O estudo sobre a estimação da função demanda de importação no Brasil é extensa, como em Portugal (1992), Ferreira (1994), Carvalho e Parente (1999), Resende (2000) entre outros. Onde estimam a função demanda de importação total e por categoria e uso. Pretende-se neste trabalho estimar apenas a função demanda de importação de bens intermediário, utilizando-se de métodos econometricos que possibilite obter resultado satisfatório. O período estende-se do primeiro trimestre de 1995 ao segundo trimestre de 2008. Período no qual o Brasil passou por uma série de mudanças de política econômica devido a alterações profundas nas condições macroeconômicas da economia.
Principalmente depois de 1999, que começa com uma mudança da taxa de cambio fixo para cambio flutuante, junto com uma crise energética que vai de 1999 a 2001, que implicou um crescimento de cerca de $3,8 \%$ a.a da economia. Em seguida o período de 2002, foi marcado pelo fim da crise energética e a crise eleitoral do fim do ano com um crescimento médio de cerca de $4.5 \%$ a.a. O que se observa nesses períodos são impactos negativos nas importações, mas com rápida recuperação.

Em geral, pode-se dizer que esse período (pós-1999), foi marcado por um ambiente favorável as importações, momentos que a taxa de câmbio não era favorável, o bom desempenho da utilização da capacidade instalada e atividades produtivas afetava as importações de forma 
positiva. Que pode ser destacado o período do fim de 2003 ao fim de 2005.

Baseando-se nesse conhecimento, este trabalho busca estimar uma função de demanda de importação de bens intermediário aplicando o mecanismo de correção de erro seguindo a metodologia de Engle e Granger (1989). E então testa se as datas acima ou a existência de outras, pode ser entendida como uma quebra estrutural, aplicando teste de Chow de quebra estrutural, CUSUM, CUSUMQ e teste recursivo sobre os resíduos. E no caso de existência ser modelada utilizando variáveis dummies. Agindo dessa forma testar se as variáveis que depende a demanda de importação têm relação uma esperada, isto é, a relação negativa entre importação e taxa de câmbio, assim como o efeito positivo da utilização da capacidade instalada e da renda nacional como encontrados em trabalhos anteriores. E verificar se em valor as elasticidade das variáveis estão muito distantes desses trabalhos realizados anteriormente.

A fundamentação teórica da função demanda de importação adotada neste trabalho é semelhante a analisada por Fachada (1990), Portugal (1992), Ferreira (1994), Alexandre e Marco (1997), Resende (1997), entre outros. Que adotam diferentes combinações da seguinte especificação:

$\ln M=a_{0}+a_{1} \ln \left[\left(e . P_{m}\right) / P_{d}\right]+a_{2} \ln Y+a_{3} \ln U+\mathcal{E}$

em que $\ln$ é a função logarítmica; $a_{0}$ é uma constante; $a_{1}$ e $a_{2}$ são as elasticidade-preço e renda da demanda de importações, respectivamente; $a_{3}$ é o coeficiente do nível de utilização da capacidade instalada; $M$ são as importações; $e$ é a taxa de câmbio nominal; $P_{m}$ é o preço externo; $P_{d}$ é o preço interno; $Y$ é o nível do produto real; $U$ é a utilização da capacidade instalada; e $\varepsilon$ um distúrbio aleatório.

A qual neste trabalho, como em Portugal (1992), assume que: o Brasil é um país de pequeno porte, de forma que aumento nas importações brasileiras não seja significativo para atingir restrições de seus fornecedores, os preços no mercado internacional não são afetados pelas ações brasileiras. Assim aceita curva de oferta completamente elástica; modelos de substituição imperfeita e homogeneidade entre preços e tarifas; ausência de ilusão monetária; e exogeneidade estrita das variáveis do lado direito das equações.
O trabalho será desenvolvido em quatro capítulos, a partir desta introdução. No capítulo 2 relata a metodologia a ser utilizada nos processos de estimação. O terceiro capítulo mostra os resultados obtidos a partir do modelo econométrico, os testes realizados $\mathrm{e}$ as elasticidades encontradas e modelo mais adequado. $\mathrm{O}$ quarto capítulo contém os comentários finais do trabalho (conclusão).

\section{2- METODOLOGIA}

\section{1- Teste de Raiz unitária}

$\mathrm{Na}$ utilização de series temporais em modelos de estimação, deve ter em mente a sua ordem de integração, ou seja, identifica quantas vezes uma série precisa ser diferenciada para se torna estacionaria. Apenas a inspeção gráfica de uma série raramente permite distinguir seu comportamento. E muitas vezes agindo assim incorre-se em equívocos. Como forma de resolver estes problemas foram desenvolvidos testes que permitem de forma mais precisa analisar o comportamento de uma série. Onde os mais tradicionais são: Dickey-Fuller (DF), DickeyFuller Aumentado (ADF) e de Phillps e Perrón (PP).

O teste de Dickey - Fuller se baseia no seguinte modelo de regressão:

$$
\Delta y_{t}=\alpha y_{t-1}+\varepsilon_{t}
$$

em que se define $\alpha=\beta-1$

$y_{t}$ denota a variável dependente e $\Delta$ denota o operador de diferença $\left(\Delta y_{t}=y_{t}-y_{t-1}\right)$. As hipóteses testadas nesse modelo correspondem a uma hipótese nula de que a série não é estacionária $\left(\mathrm{H}_{0}: y_{t}\right.$ não é I (0) ou $\left.\alpha=0\right)$; contra a hipótese alternativa de que a série não é integrada, ou seja, trata-se de uma série estacionária $\left(\mathrm{H}_{1}: y_{t}\right.$ é $\left.\mathrm{I}(0)\right)$.

O problema desse teste é que considera os erros como sendo ruído branco. Mas nem sempre isso ocorre (pode ter autocorrelação). Uma saída pode ser incorporar na equação (2) valores defasados da variável endógenas $\left(y_{t}\right)$ a fim de eliminar a presença

${ }^{1}$ Sem constante e sem tendência. 
De autocorrelação entre os termos de erros, ou seja,

$$
\Delta y_{t}=\alpha y_{t-1+} \sum_{i=1}^{p-1} \lambda_{i} \Delta y_{t-1} \mathcal{E}
$$

Sendo

$$
\lambda_{i}=-\sum_{j=i+1}^{p} p_{i}
$$

Em que $p$ é a ordem do modelo autoregressivo ou o número de defasagens suficientes para que os resíduos resultantes sejam não correlacionados (ruído branco). Com essa nova versão o teste recebeu o nome de Dicky-Fuller Aumentado.

O número de defasagem a colocar deve ser de tal forma que os resíduos estimados sejam ruído branco.Outra possibilidade equivalente é selecionar o numero de defasagem através dos critérios de informação AIC (critério de informação Akaike) e o critério de Schwarz. Isto é, deve-se escolher o valor da defasagem que resulta no menor Akaike ou Schwarz e confirmar o resultado verificando se os resíduos comportamse como um ruído branco.

O critério de informação de Akaike (AIC) pode se definido como:

$$
A I C=\ln \left({\sigma_{e}}^{2}\right)+2 \cdot n / T,
$$

enquanto o critério de informação de Schwartz (SBC) é:

$$
\begin{gathered}
\mathrm{SBC}=\ln \left(\sigma_{e}\right)+[2 \cdot \ln (T) \cdot n / t], \text { onde } \\
\sigma_{e}{ }^{2}=\left(\sum \varepsilon_{t}{ }^{2}\right) / T
\end{gathered}
$$

onde $\mathrm{n}$ é o numero de parâmetros estimados e $\mathrm{T}$ é o numero de observações.

A diferença entre eles é que o critério de informação de Schwartz penaliza mais que o critério de informação de Akaike em modelos que utiliza muitos parâmetros, pois $\operatorname{lnT}>2$ para amostras com $\mathrm{T} \geq 8$.

O outro teste de raiz unitária é o de Phillips-Perron, que faz uma correção não paramétrica ao teste de Dicky-Fuller, permitindo que seja consistente mesmo que haja variáveis dependentes defasadas e correlação serial nos erros. Isto se deve ao fato de quando se tem menos de 50 observações, colocar mais parâmetros, como faz o teste $\mathrm{ADF}$, leva a perda de potência do teste.

\subsection{Teste de cointegração}

Trabalhar com séries em níveis em uma regressão, embora permita captar as relações de longo prazo entre as variáveis, pode produzir ao que se convencionou chamar de regressão espúria. Isto é, ao estimar uma variável contra a outra, terá alta correlação, um $\mathrm{R}^{2}$ e teste $\mathrm{t}$ significativo, quando na verdade não deveria ser. Isso deve-se exclusivamente ao fato de ambas apresentarem tendência ao longo do tempo e não a uma relação econômica entre elas.

Uma solução inicialmente sugerida para este problema foi a de usar as variáveis em primeiras diferenças, o que eliminava sua tendência. Em que se baseava no fato de que se as variáveis possuem realmente uma relação, suas taxas de variações também possuirão. Mas acontece que agindo dessa forma as variáveis perdem importantes relações de longo prazo.

Portanto uma forma de contornar este problema foi desenvolvida por Engle e Granger (1987), em que é possível trabalhar com o nível das séries sem correr o risco de regressões espúrias e isso ocorre quando as séries são cointegradas. Dessa forma a análise de cointegração, foi estabelecida como um pré-teste para evitar problemas de regressão "sem sentido".

O conceito de cointegração vem de: se uma série precisa ser diferenciada $\mathrm{d}$ vezes antes de tornar-se estacionária, então ela contém d raízes unitária e é dita ser integrada de ordem d, denotada por I (d). Se considerarmos duas séries de tempo $y_{\mathrm{t}}$ e $x_{t}$, ambas integrada de ordem d, em geral, uma combinação linear dessas séries será também I(d). Contudo, poderá existir uma combinação das séries $\mathrm{I}(\mathrm{d})$ que seja $\mathrm{I}(\mathrm{d}-\mathrm{b})$ com b $>0$. E então diz que as séries são cointegradas.

Supondo $d=b=1$. Temos:

$$
x_{t} \sim I(1) \text { e } y_{t} \sim I(1)
$$

mas,

$$
Z_{t}=\left(y_{t}-\alpha x_{t}\right) \sim I(0)
$$


Onde $\alpha$ é o vetor de cointegração. Dizemos que a série é CI $(1,1)$.

Assim para estimar a relação de equilíbrio de longo prazo entre as variáveis é necessário apenas estimar o modelo por mínimos quadrados ordinário (como apresentado abaixo nos dois estágios), em uma regressão em nível e aplica o teste de raiz unitária sobre os resíduos dessa regressão, sendo consideradas as séries cointegradas aquelas variáveis cuja séries dos resíduos seja estacionaria em nível. Para verificar a estacionariedade das séries dos resíduos aplicas os teste de raiz unitária citados acima utilizando valores críticos tabelados, por exemplo por Macnnon (1991).

Caso os resíduos sejam estacionários, tem-se a indicação de que as séries analisadas possuem uma relação de longo prazo (cointegra) e de que existe um modelo de correção de erro (MCE), que permite obter a relação de curto prazo entre as variáveis (se elas tem realmente uma relação de longo prazo, então é possível haver desequilíbrios de curto prazo).

Para a estimação Engle e Grander (1987), sugeriram a utilização de um método de dois estágios ao se lidar com o mecanismo de correção de erros. Que implica na seguinte forma:

1- No primeiro estágio, o vetor de cointegração, contendo as relações de longo prazo, é estimado através de uma regressão estática em nível.

2- $\quad$ No segundo estágio, o termo de correção e erro, isto é, o resíduo obtido no primeiro estagia, é usado em uma equação em diferenças para se obter os coeficientes de impacto.

Em outras palavras, no primeiro estágio, usamos as séries em I(d) para extrair as informações de longo prazo e ignoradas as considerações sobre a dinâmica. A estrutura dinâmica é incorporada apenas no segundo estágio quando as variáveis aparecem na forma de diferenças, enquanto as restrições em nível do primeiro estagio são incorporadas através do termo de correção de erro.

Uma observação que deve ser feita, como notado por Banerjee (1993) é que para pequenas amostras é comum no método de Engler e Granger (1987) os resíduos de uma regressão estática sejam mal comportado, o que pode levar ao reconhecimento de cointegração espúria, ou a ignora as relações de longo prazo.

Stock (1987) citado por Portugal (1992), no método de Engler Granger (1987), encontrou em pequenas amostras evidências de viés relativamente grande. Que é relevante em regressões de cointegração com $\mathrm{R}^{2}$ baixo.

A análise de quebra estrutural apresentada neste trabalho será com base no teste de Chow, CUSUM e CUSUMQ, e não será apresentado aqui no intuito de resumir, um estudo sobre eles encontra-se em CUTHBERTSON, HALL e TAYLOR (1992. cap.4). Da mesma forma que a metodologia de Johansen (1990), não será apresentada e pode ser encontrada em diversos livros e artigos, como o de Portugal (1992) e do próprio Johansen (1990).

\section{3 - RESULTADOS EMPIRICO}

Todas as especificações utilizadas neste trabalho apresentam dados com periodicidade trimestral, sem ajuste sazonal, e abrangem o período entre o primeiro trimestre de 1995 ao segundo trimestre de 2008. Sendo as importações de bens intermediários, série de comércio exterior da FUNCEX; taxa de câmbio real foi calculada usando a taxa de cambio média, IPCA e IPC USA (CPIAUCNS); PIB fornecido pelo IBGE; e a utilização da capacidade instalada fornecido pela FGV/DADOS. As séries foram transformadas por intermédio da função logaritma, o que permite a obtenção direta das elasticidades e reduz a variabilidade das séries. Novamente em relação à periodicidade das estimações, todas as séries disponíveis, com exceção do PIB nacional e da taxa de utilização da capacidade instalada, são mensais. Então foram trimestralizada a partir de uma média aritmética simples.

Para análise das variáveis utilizou-se o pacote econometrico EVIEWS 6.0.

\subsection{Comportamento das series:}

Primeiramente são apresentados os gráficos que descrevem o comportamento das séries (em níveis) utilizadas na estimação da função demanda de importação de bens intermediário em (logaritmo): 

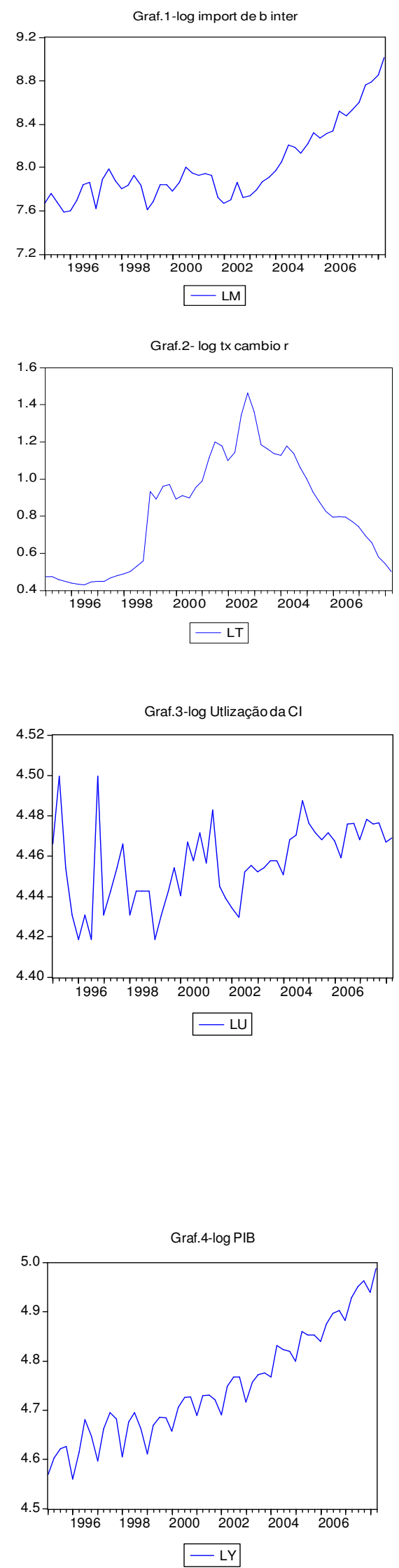

Gráficos de séries em primeira diferença:
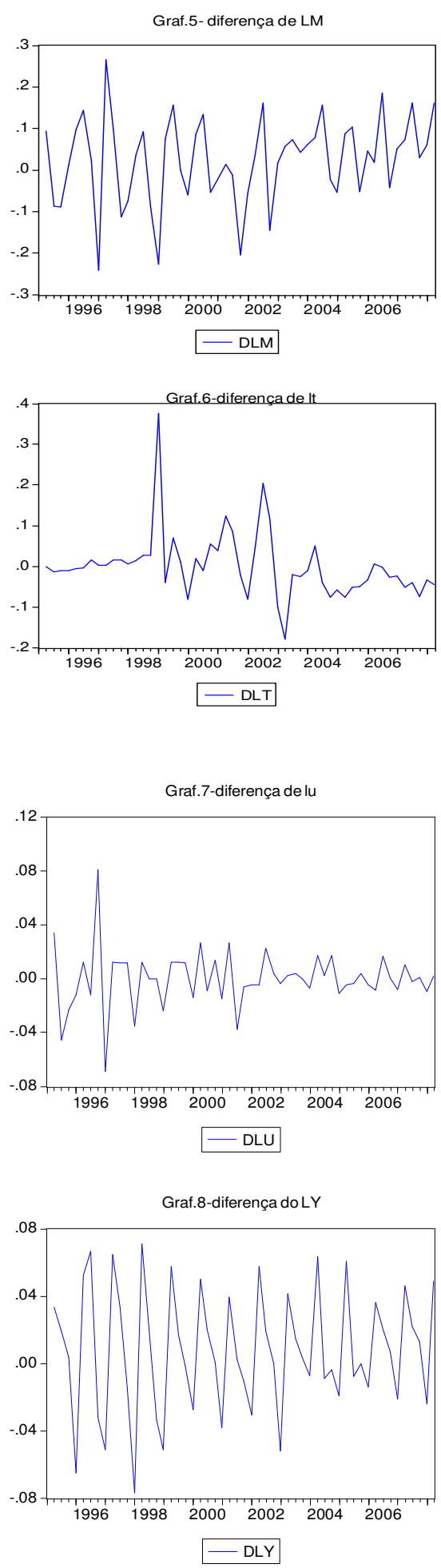

O gráfico 1 mostra a evolução das importações brasileira de bens intermediário do primeiro trimestre de 1995 ao segundo trimestre 
de 2008. Onde algumas perturbações ao longo da trajetória da serie se deve a alterações da política macroeconômica, como exemplo, a mudança na política cambial ocorrida no primeiro trimestre de 1999, que se observa no gráfico 2, pode ter afetado a redução das importações neste período como indicada no gráfico 1 . Assim como a crise no setor enérgico por volta de 2001 (racionamento de energia com a economia crescendo entorno de $3.8 \%$ a.a), e as eleições ocorrida em 2002 (com crescimento médio anualizado de $4.5 \%$ a.a). Todos eles são possíveis fatores capazes de provocar mudança no comportamento das importações.

Pode-se dizer que a partir de 2002 até 2008, o aumento das importações manteve uma relação crescente devido o ambiente favorável. Como pode ser visto nos gráficos 2,3 e 4 , em geral, a desvalorização da taxa de câmbio ${ }^{2}$, como o aumento da capacidade instalada no setor de bens intermediário e aumento na renda nacional pode ter sido os principais fatores o responsáveis por seu crescimento.

A visualização dos gráficos $1,5,4$ e 8 e teste gráfico obtido através do pacote do EVIEWS como apresentado em 9 e 10, permite detectar períodos de sazonalidade na série de importação e do PIB. Neste caso pode tanto dessazonalizar utilizando método X11 e X12, ou como faz Carvalho e Parente (1999), na estimação incluir dummies sazonais.

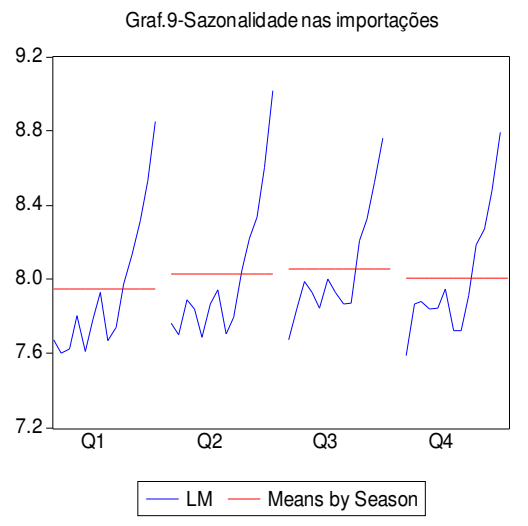

\footnotetext{
2 Uma observação que deve ser feita é que entorno do período de 1999 até 2003, como mostra o gráfico 1, mesmo com cambio desfavorável as importações se manteve alta, que pode ser explicada pelo bom comportamento das outras variáveis.
}

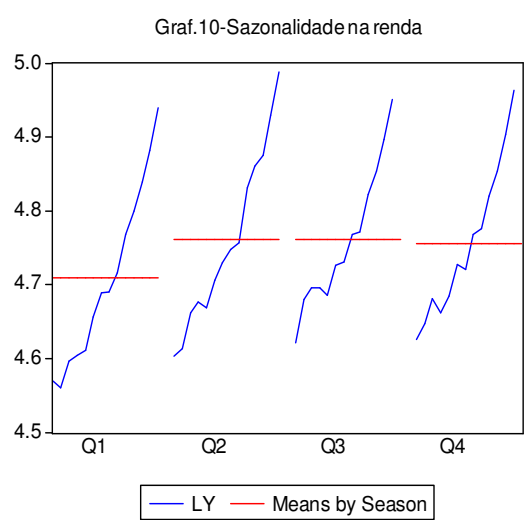

Porém este trabalho, assim como em Portugal (1992), não será feito ajuste sazonal. Seguindo a mesma idéia de que grande parte do comportamento sazonal nas séries de importação como mostra os gráficos, parece ser explicada pela sazonalidade na variável renda nacional.

\subsection{Teste de raiz unitária:}

A inspeção dos gráficos acima em níveis e em diferenças permite ter uma idéia se a série é estacionaria em nível ou em diferença, ou em nenhuma das duas. Mas acontece que nem sempre isso é possível, segundo Hendres (1995), muitas vezes agindo assim pode incorrer em equívocos. Dessa forma partimos para os tradicionais teste de raiz unitária DF, DFA e PP, sobre cada uma das variáveis, cujos resultados estão apresentados na tabela $1 \mathrm{em}$ anexo. Como apresentado o número de defasagem a colocar foi escolhido de tal forma que os resíduos estimados sejam ruído branco. E outra possibilidade equivalente é através dos critérios de informação AIC (critério de informação Akaike) e o critério de Schwarz. Isto é, deve-se escolher o valor da defasagem que resulta no menor Akaike ou Schwarz e confirmar o resultado verificando se os resíduos comportamse como um ruído branco.

O teste foi realizado com intercepto, com intercepto e tendência, sem intercepto e sem tendência. Portanto, a literatura implicada que usar ou não estes parâmetros depende muitas vezes de qual série esta utilizando, a sua análise gráfica pode ser utilizada como ferramenta. Além da existência de teste para provar a sua significância.

A série da utilização da capacidade instalada considerando o testes de ADF com intercepto e sem intercepto e no teste de PP sem 
intercepto é integrada de ordem um. E dessa forma todas as séries apresentam raiz unitária em nível, mas quando é aplicado a primeira diferenças mostrou ser estacionaria, isto é, todas as séries são integradas de ordem um (I (1)). O que satisfaz a primeira condição para aplica o método de cointegração entre as variáveis utilizadas.

\subsection{Análise de cointegração}

Como propõe o método Engle e Granger (1987), satisfeita a primeira condição para uma possível relação de longo prazo entre as variáveis, isto é, todas são integradas de ordem de mesma ordem. Então estimou-se uma regressão estática do logaritimo da demanda de importação de bens intermediário contra o logaritimo da taxa de câmbio real, da utilização da capacidade instalada e da renda nacional. Da seguinte forma:

$\ln Q_{t}=\alpha_{0+} \alpha_{1} \ln T_{t+} \alpha_{2} \ln U_{t+} \alpha_{3} \ln Y_{t+} \varepsilon_{t}$ (7)

Onde, $Q_{t}$ são as importações de bens intermediário no tempo t; $T_{t}$ é a taxa de cambio real em t; $U_{t}$ é a utilização da capacidade instalada em t; $\quad Y_{t}$ é a renda nacional em t; e $\mathcal{E}_{t}$ é o termo de erro aleatório. ln é o logaritmo natural na tabela 1 :

Os resultados obtidos estão apresentados

TABELA 1: Estimação do vetor de longo prazo para a demanda de bens intermediários

\begin{tabular}{ccccc}
\hline Variáveis & Coef. & DP. & t-estat. & Prob. \\
\hline$l t_{t}$ & -0.392 & 0.038 & -10.417 & 0.000 \\
$l u_{t}$ & 1.439 & 0.663 & 2.168 & 0.035 \\
$l y_{t}$ & 3.0283 & 0.121 & 24.979 & 0.000 \\
Const. & -12.454 & 2.691 & -4.629 & 0.000 \\
\hline \multirow{2}{*}{$\mathrm{R}^{2}=0.95$} & $\mathrm{DW}=$ & $\mathrm{DF}=-$ & $\mathrm{ADF}=-$ & $\mathrm{PP}=-$ \\
& 1.71 & $2.37^{*}$ & $2.57^{<}$ & 6.35 \\
\hline
\end{tabular}

Fonte: elaboração própria. ${ }^{<}$Teste realizado sem tendencia e sem intersepto (tendência e intersepto não foram significativa) baseado nos teste convencionais. ${ }^{*}$ aceita hipotese nula a $1 \%$ de significância; $\operatorname{lm}$ - $\log$ das importações de bens intermediário; lt- $\log$ da taxa de cambio real; lu - log da utilização da capacidade instalada; ly - log do PIB.

Como $l m_{t}, l t_{t}, l u_{t}$ e $l y$, são individualmente não estacionária, existe como dito acima, uma possibilidade de que essa regressão seja espúria, sem sentido. Para solucionar este problema proceder como: aplicar os teste tradicionais de raiz unitária sobre os resíduos obtido da regressão
1; obter que os resíduos são I (0); isto é são estacionários (pelos testes tradicionais citados). Esta não é a forma apropriada usada para trata este caso. Segundo Engle e Granger (1989), como os resíduos são estimados, não é possível usar os valores tabulados convencionalmente. Portanto, trata-se, agora, de usar uma tabela apropriada a variáveis estimadas, como a encontrada em seu trabalho. De outra forma pode-se utilizar o procedimento de Macknnom (1991).

Utilizando a tabela desse último, que esta apresentada em anexo. Encontrou-se que pelos testes de Dickey e Fuller, e Dickey e Fuller Aumentado, a não rejeição da hipótese nula de raiz unitária. Mas o teste de Phillps-Perron, tanto com intercepto e com intercepto e tendência rejeitou a hipótese nula de raiz unitária. Como implica a literatura, verificar a existência de raiz unitária quando se tem uma amostra pequena, o teste de Phillps-Perron é mais apropriado que o Dickey e Fuller Aumentado. Tendo em vista que dispomos de uma amostra pequena neste trabalho, seguiremos o resultado do teste de Phillps-Perron e conclui-se que os resíduos estimados são estacionários.

Por conseguinte, a equação é uma regressão co-integrante e não espúria, ainda que individualmente as variáveis sejam não estacionárias. Dessa forma pode denomina a regressão como uma função demanda de importação estática ou de longo prazo e interpretar seus coeficientes como sendo de longo prazo.

Os sinais dos coeficientes obtidos estão de acordo com esperado, com impacto negativo da taxa de câmbio de -0.39 e a influência positiva da renda nacional e da utilização da capacidade instalada sobre a demanda de importação, de 1.44 e 3.03, respectivamente. Mas os valores das elasticidades diferenciam dos resultados encontrados na literatura, como em Portugal (1992) para o período de 1975 a 1987 com elasticidade do câmbio, renda e utilização da capacidade instalada, de -0.908, 0.972, 3.672, respectivamente. Assim como em Fachada (1990), citado por Portugal (1992), que apresentou valores de $-0.87,1.16$ e 2.88 , para o período de 1976/88, em Parente e Carvalho (1999) no período de $1978 / 1996$ foi de $-2.00,1.34$ e 0.77 .

Essa diferença pode ser devido, em alguns períodos pós-1999 (mais ou menos entre 2002 e 2004), mesmo com a taxa de câmbio 
valorizada, as importações se mantiveram em crescimento, explicado pelo bom desempenho da atividade produtiva. Obtido a relação de longo prazo entre as variáveis sem o problema de regressão espúria então, pelo Teorema de representação de Engle e Granger (1987), existe um mecanismo de correção de erro para tratar das relações entre as variáveis. Ou seja, se há uma relação de longo prazo entre asvariáveis, é possível que no curto prazo haja um desequilíbrio.

Dessa forma pode usar o termo de erro para ligar o comportamento de curto prazo da variável com seu valor de longo prazo.

TABELA 2: Teste de Dickey-Fuller

\begin{tabular}{c|cc|c}
\multirow{2}{*}{ Variável } & \multicolumn{2}{|c|}{ DF } & \multirow{2}{*}{ Integração } \\
\cline { 2 - 4 } & $\mathrm{C}$ & $\mathrm{C} \mathrm{e} \mathrm{T}$ & \\
\hline Lm & 0.921786 & -0.950091 & $\mathrm{I}(1)$ \\
Dlm & -2.015175 & -2.491 .265 & $\mathrm{I}(0)$ \\
$\mathrm{Lt}$ & -0.849343 & -0.419785 & $\mathrm{I}(1)$ \\
Dlt & -5.618001 & -5.969791 & $\mathrm{I}(0)$ \\
Lu & $-3.025983^{\wedge}$ & $-3.928837^{\wedge}$ & $\mathrm{I}(0)$ \\
Ly & $2.220672^{*}$ & -1.059988 & $\mathrm{I}(1)$ \\
Dly & -6.875465 & -2.357208 & $\mathrm{I}(0)$
\end{tabular}

Fonte: elaboração própria.* - aceita hipótese nula a $1 \%$ de significância; ${ }^{\wedge}$ - estacionaria em nível; $\mathrm{C}$ - constante; T tendência; $\operatorname{lm}$ - log das importações de bens intermediário; dlm- primeira diferença de $1 \mathrm{~m} ; 1$ - log da taxa de cambio real; lu - log da utilização da capacidade instalada; ly - log do PIB; dly - primeira diferença de ly; dlt - primeira diferença de lt; dlu - primeira diferença de lu.anteriores.
Procedendo assim estimou-se uma equação em diferenças mais geral (apresentada em anexo) defasadas e o termo de erro da regressão cointegrante defasado em um período (vetor de correção de erro) e testou seus coeficientes, sendo eliminados aqueles não significativos e optandose por aqueles que apresentassem menor critério de informação Schwarz e Akaike, com resíduos mais bem comportados. A equação que apresentou mais adequada esta na tabela 3 .

TABELA 3: Mecanismo de correção de erro para a demanda de bens intermediário

\begin{tabular}{ccccc}
\hline \hline Variáveis & Coef. & DP & $\begin{array}{c}\mathrm{t}- \\
\text { Statistic }\end{array}$ & Prob. \\
\hline DLT & -0.229 & 0.118 & -1.941 & 0.0581 \\
DLU & 1.448 & 0.469 & 3.089 & 0.0033 \\
DLY & 1.894 & 0.297 & 6.375 & 0.0000 \\
$\mathrm{C}$ & 0.009 & 0.009 & 0.912 & 0.3662 \\
$\mathrm{RE} 1 \mathrm{O}$ & -0.656 & 0.137 & -4.789 & 0.0000 \\
\hline $\mathrm{R}^{2}=0.62$ & $\mathrm{DF}=-3.33$ & $\mathrm{ADF}=-3.28^{*}$ & $\begin{array}{c}\mathrm{PP}=- \\
7.26\end{array}$ & $\mathrm{DW}=$ \\
& & & White $=$ \\
AIC $=-$ & $\mathrm{SBC}=-$ & $\mathrm{LM}=2.66$ & 0.76 \\
2.45 & 2.26 & Valor $\mathrm{p}=0,08$ & $\begin{array}{c}\text { valor } \\
\mathrm{p}=0,69\end{array}$ \\
& & & & \\
\hline
\end{tabular}

Fonte Elaboração própria: DLT - diferença da taxa de cambio; DLU- diferença da utilização da capacidade instalada DLY - diferença da renda nacional; C- constante; RE1O - resíduo da regressão co-integrante defasado;DWDurbin -Watson
TABELA 4: Teste Phillips-Perron

\begin{tabular}{c|c|c|c|c}
\hline \multirow{2}{*}{ Variável } & \multicolumn{3}{|c|}{ PP } & Integração \\
\cline { 2 - 4 } & $\mathrm{C}$ & $\mathrm{C}$ e T & $\mathrm{S}$ & \\
\hline $\mathrm{dm}$ & $2.90^{*}$ & -0.586 & 1.947 & $\mathrm{I}(1)$ \\
$\mathrm{dlm}$ & -7.14 & -1.337 & -6.834 & $\mathrm{I}(0)$ \\
$\mathrm{lt}$ & -1.14 & -0.105 & -0.296 & $\mathrm{I}(1)$ \\
$\mathrm{dlt}$ & -5.53 & -5.82 & -5.594 & $\mathrm{I}(0)$ \\
$\mathrm{lu}$ & - & - & 0.071 & $\mathrm{I}(1)$ \\
$\mathrm{dlu}$ & $4.61 \#$ & $5.627 \#$ & & \\
$\mathrm{ly}$ & -0.06 & - & -1.355 & $\mathrm{I}(0)$ \\
$\mathrm{dly}$ & -1.34 & -2.155 & -8.446 & $\mathrm{I}(1)$ \\
\hline
\end{tabular}

Fonte: elaboração própria. Idem variáveis anteriores.

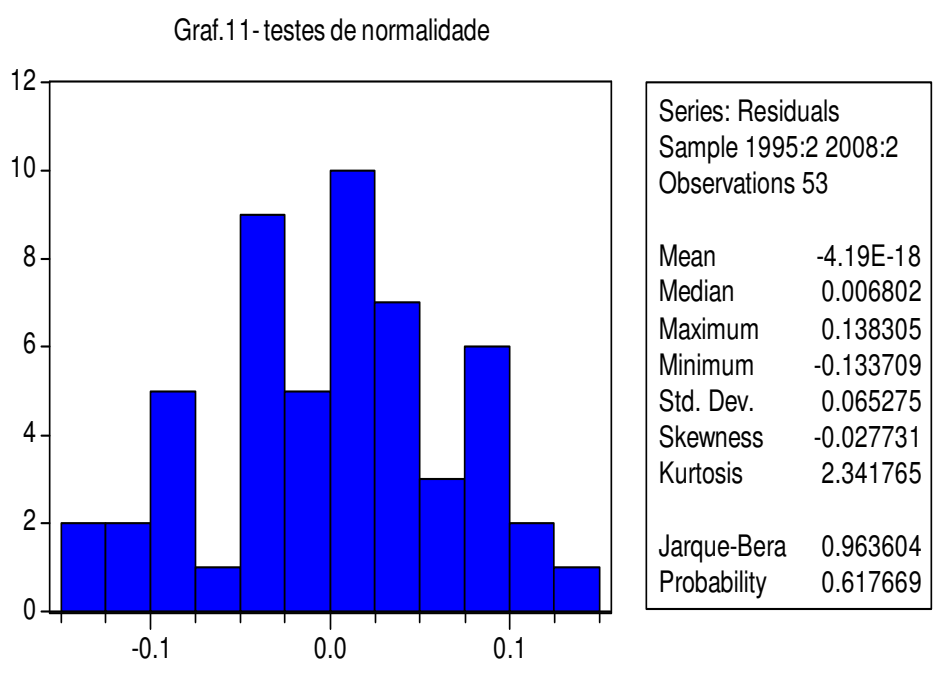

391

Revista da Universidade Vale do Rio Verde, Três Corações, v. 10, n. 1, p. 384-395, 2012 
TABELA 5: Teste de Dickey-Fuller Aumentado

\begin{tabular}{c|c|c|c|c}
\hline \multirow{2}{*}{ Variável } & \multicolumn{3}{|c|}{ ADF } & \multirow{2}{*}{ Integração } \\
\cline { 2 - 4 } & $\mathrm{C}$ & $\mathrm{C} \mathrm{e} \mathrm{T}$ & $\mathrm{S}$ & \\
\hline lm & 208.277 & 0.31553 & 253.117 & $\mathrm{I}(1)$ \\
Dlm & -7.440 .421 & -8.141 .059 & -6.700 .505 & $\mathrm{I}(0)$ \\
Lt & -1.030 .053 & -0.087487 & -0.296674 & $\mathrm{I}(1)$ \\
Dlt & -5.567 .432 & -5.938 .090 & -5.623 .275 & $\mathrm{I}(0)$ \\
lu & $-3.294048^{*}$ & $-3.840074^{\#}$ & -0.235257 & $\mathrm{I}(1)$ \\
dlu & -1.207 .750 & -1.206 .648 & -1.219 .179 & $\mathrm{I}(0)$ \\
ly & 1.425 .106 & -0.248349 & 3.165 .559 & $\mathrm{I}(1)$ \\
dly & $-3.182270^{* *}$ & $-3.56994^{* *}$ & -8.750 .786 & $\mathrm{I}(0)$ \\
\hline
\end{tabular}

Fonte: elaboração própria. Idem variáveis tabela

Os resultados apresentam-se bastante razoáveis com sinais esperados. $\mathrm{O}$ teste de multiplicador de lagrange e Durbin-Watson não rejeita a hipótese nula de que não há autocorrelação serial. E o teste White não rejeita a hipótese de que não há heterocedasticidade nos resíduos aos níveis de significância de 1\%, 5\% e 10\% (valores críticos: $6.25,7.81,9.34$ ). Além desses aplicou-se o teste de Jaque-Bera mesmo considerando o tamanho da amostra pequeno, como apresentado a seguir:

Verifica-se que a estatística JB é de cerca de 0,97 e que a probabilidade de se obter-se esse número, pressupondo-se uma distribuição normal, é de cerca de $62 \%$. Portanto, não deve rejeita a hipótese nula de normalidade dos resíduos. Porém, como observado acima deve ter cuidado com o tamanho da amostra, uma vez que o teste requer grande amostras.

Além da estimação utilizando o teste de Engler e Granger, realizou-se o teste de cointegração de Johansen, o qual esta apresentado em apêndice. Que se aplica da seguinte forma:

Como todas as séries possuem uma raiz unitária, portanto são I (1) e esta não estacionariedade das séries indica que é possível realizar através do procedimento de Johansen, assim como o teste de Engler e Granger, o teste de co-integração.

O primeiro resultado fornecido pelo teste de Johansen é o número de vetores de cointegração. Para isso são utilizados os testes do traço e do máximo autovalor, cujos resultados estão expostos na Tabela em apêndice. Ambos os testes indicam a existência de apenas um vetor de co-integração.

Para verificar a robustez e o ajustamento do modelo que foi utilizado no procedimento de Johansen, realizou-se o teste de autocorrelação entre os resíduos. O modelo adequado é aquele que não possui seus resíduos autocorrelacionados (Ender, 2003).

O resultado do teste de co-integração indica que há uma relação de equilíbrio entre as séries de importações de bens intermediário, taxa de câmbio real, utilização da capacidade instalada e o PIB, embora ambas individualmente sejam não estacionárias.

Portanto, notou-se, assim como em Portugal (1992), a não evidência de vetores multiintegrados, e por isso, se matem a metodologia/resultados de Engler e Granger (1987) apresentada acima.

\section{4- Análise de Quebra estrutural}

Devidos as mudanças exógenas ocorridas ao longo do período, faz necessária a aplicação de testes que visem identificam possíveis quebras estruturais no modelo, uma vez que isso ocorra, deve ser tratado com cuidado. Pois os parâmetros passam a ser instáveis e se tratados como estáveis provavelmente chegará a resultados errôneos. Para a sua verificação aplicou os seguintes testes abaixo:

Teste Chow de quebra estrutural:

A idéia do teste é calcular as regressões separadamente para cada subamostra e verificar se a diferença significativa nas equações estimadas. Uma diferença significante indica uma mudança estrutural. A estatística F para o teste, gerada pelo Eviews, se baseia na comparação da SQR (soma dos quadrados dos resíduos) restrita e irrestrita, sob a hipótese nula de ausência de mudança estrutural.

Então aplicou-se o teste até o primeiro trimestre de 2003 (período de prováveis quebra, devido mudança na política econômica), temos: 
TABELA 6: Teste de quebra estrutural de Chow

\begin{tabular}{llllll}
\hline \hline Ano & $\begin{array}{l}\text { F- } \\
\text { estatist }\end{array}$ & $\begin{array}{l}\text { Valor- } \\
\mathrm{p}\end{array}$ & Ano & $\begin{array}{l}\text { F- } \\
\text { estatist }\end{array}$ & $\begin{array}{l}\text { Valor- } \\
\mathrm{p}\end{array}$ \\
\hline 1998:01 & 1.570 & 0.188 & $2000: 03$ & 0.533 & 0.749 \\
\hline 1998:02 & 0.640 & 0.670 & $2000: 04$ & 0.434 & 0.822 \\
\hline 1998:03 & 0.677 & 0.642 & $2001: 01$ & 0.433 & 0.822 \\
\hline 1998:04 & 0.678 & 0.642 & $2001: 02$ & 0.210 & 0.956 \\
\hline 1999:01 & 0.821 & 0.541 & $2001: 03$ & 0.376 & 0.861 \\
\hline 1999:02 & 1.018 & 0.418 & $2001: 04$ & 0.588 & 0.708 \\
\hline 1999:03 & 0.960 & 0.452 & $2002: 01$ & 0.334 & 0.889 \\
\hline 1999:04 & 0.890 & 0.495 & $2002: 02$ & 0.493 & 0.779 \\
\hline 2000:01 & 0.675 & 0.644 & $2002: 03$ & 0.272 & 0.925 \\
\hline 2000:02 & 0.467 & 0.798 & $2002: 04$ & 0.435 & 0.821 \\
\hline 2000:03 & 0.533 & 0.749 & $2003: 01$ & 0.454 & 0.807 \\
\hline
\end{tabular}

Fonte: Elaboração Própria.

Como pode ser visto na tabela 6 , em ambas as datas, tem-se um F-estatístico menor que o $\mathrm{F}$ crítico aos níveis de significância de $1 \%, 5 \%$ e $10 \%$; e valor-p elevado.E dessa forma, é possível concluir que não há mudança significativas nos parâmetros, isto é, não rejeita a hipótese nula de estabilidade dos parâmetros.

Partiu-se para a estimação recursiva:

a - Teste recursivo sobre os resíduos: A hipótese nula é de que o vetor de coeficientes é o mesmo para todo o período. A presença de resíduos fora do intervalo de +/- 2 é um indício de instabilidade dos parâmetros, pelo gráfico abaixo se percebe uma perturbação em torno de 2008.

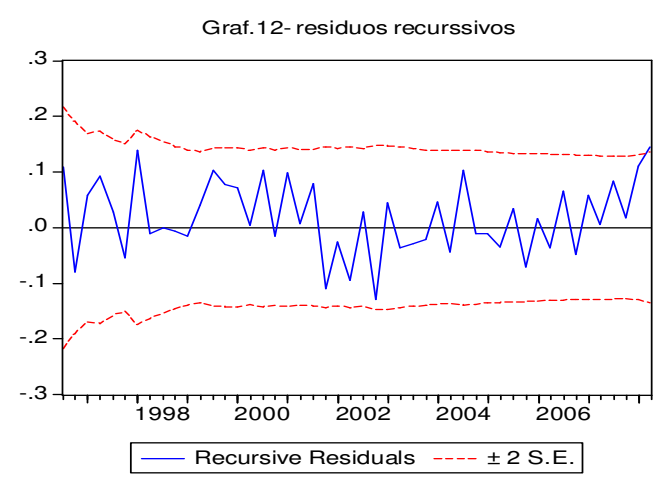

b - Teste CUSUM: se baseia na soma cumulativa dos resíduos, obteve uma pequena alteração entorno do ano de 2001.

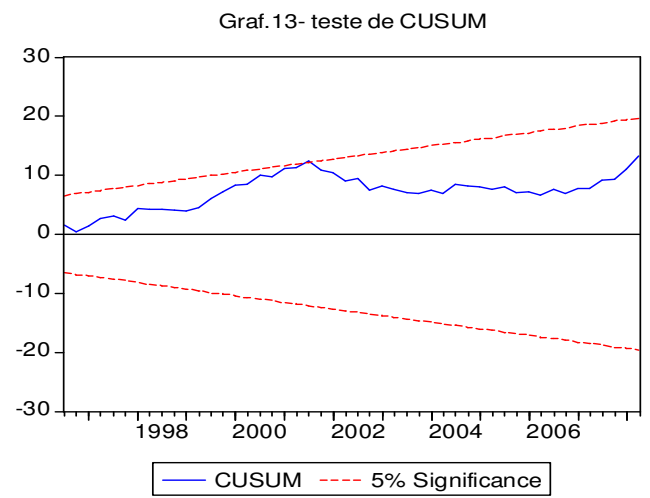

c - Teste de CUSUMQ: A diferença desse para o CUSUM é que o que acontece com o erro de previsão um passo a frente ao quadrado. Não indica alterações claras dos parâmetros.

Graf.14- teste de CUSUMQ

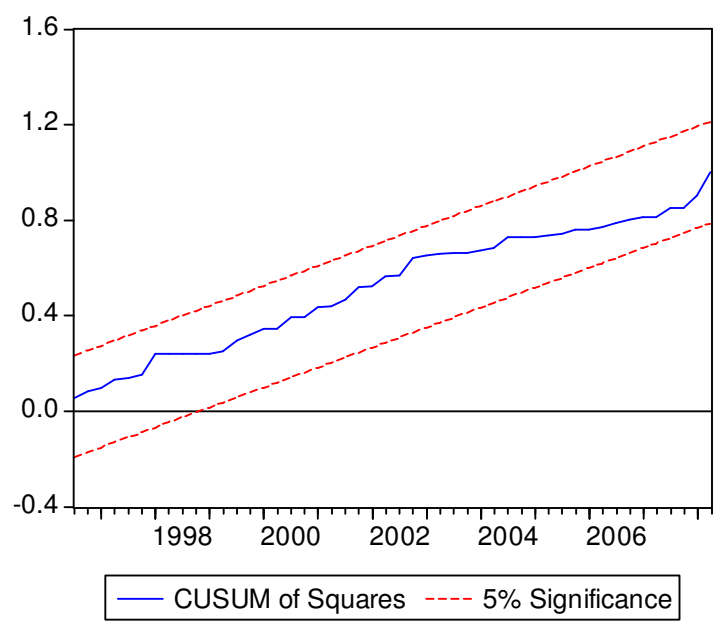

d - Teste de coeficientes recursivos: Que possibilita acompanhar a evolução das estimativas dos coeficientes a medida que a amostra evolui do mínimo necessário para o máximo possível de observações. Diz se o coeficiente não apresenta sinal de convergência à medida que mais observações são utilizadas na estimação, temos indícios de estabilidade estrutural. Neste caso, com exceção do coeficiente da utilização da capacidade instalada, todos aparentaram convergir. Os testes apresentados não apresentaram uma mudança clara no modelo a ponto de ser identificada como uma quebra estrutural. Porém análise gráfica das variáveis, 
permiti analisar possíveis flutuações exógenas no modelo. Como o período mudança de câmbio fixo para câmbio flutuante ocorrida em 1999:1; o racionamento de energia ocorrido em 2001; a eleição ocorrida em 2002. Por conveniência reestimou o modelo com dummies nessas datas e em 2008 indicada pelo teste 2 e estão apresentados em anexo. Com exceção da dummy para o ocorrido em 2002, todas foram significativas e melhores critério de informação. Mas como os testes não apresentaram grandes evidências contra a não constância das estimações ao longo do período considerado, optou-se pelo modelo sem dummies.

Assim os resultados considerados mais apropriados, são os apresentados nas tabelas 4 e 5 . A tabela 5 mostra que a variação percentual das importações de bens intermediário depende da variação percentual da taxa de câmbio, da renda e da utilização da capacidade instalada e também do termo de erro de equilíbrio. Como esperado todas as variáveis tem sinal pertinentes e coeficiente significativos. $\mathrm{O}$ sinal do parâmetro do termo de correção de erros negativo indica uma velocidade de ajuste a variações em torno do equilíbrio de longo prazo de aproximadamente $65 \%$ por período.

Em outras palavras o termo de erro, mostra que podemos afirmar que cerca de $65 \%$ da discrepância entre $o$ valor da quantidade importada de bens intermediário e o valor de longo prazo das variáveis, é eliminada ou corrigida a cada trimestre. Que é um valor relativamente alto indicando que a correção do desequilíbrio, a cada trimestre é extremamente rápida.

Em Portugal (1992) este valor foi de cerca de 54\%, Castro e Calvacanti (1997) de 36\%, Parente e Carvalho (1999), cerca de 13\%, Resende (2000) de $45 \%$. Nota-se que é um valor relativamente elevado em comparação com os antecedentes.

\section{5 - COMENTARIOS FINAIS}

Este trabalho buscou a estimação da demanda de importação de bens intermediários, a qual parte do usual na literatura, colocando-a como função da taxa de câmbio real, da renda nacional e da utilização da capacidade instalada do setor. Partindo do pressupondo que: o Brasil é um país de pequeno porte, de tal forma que aumento nas importações brasileiras não seja significativo para atingir restrições de seus fornecedores, os preços no mercado internacional não são afetados pelas ações brasileiras. Assim aceita curva de oferta completamente elástica; modelos de substituição imperfeita e homogeneidade entre preços e tarifas; ausência de ilusão monetária; e exogeneidade estrita das variáveis do lado direito das equações.

Esta se deu através da aplicação do Método sugerido por Engle e Granger (1987). Os testes de diagnóstico, como teste LM, DarbinWatson, teste de normalidade foram satisfeito. Apesar dos sinais dos parâmetros e sua significância estarem de acordo com o esperado, isto é, a relação tanto de longo prazo quanto de curto prazo está pertinente, os seus valores divergem um pouco dos trabalhos anteriores, por exemplo, em Portugal (1992), Castro e Calvacanti (1997), Parente e Carvalho (1999), Resende (2000).

Apesar desses resultados aceitáveis, alguns problemas podem surgir, pelo fato do período analisado ter passado por mudanças importantes na política macroeconomia, os testes utilizados neste caso como DF, ADF e PP de raiz unitária pode não ser apropriado, como notado por Perron (1989), assim como os testes de cointegração de Engle e Granger (1987) não ser apropriado para testar cointegração com mudança de regime devido esse teste presumir que o vetor de cointegração é invariante no tempo. Mesmo com a superconsistência da estimação via mínimos quadrados estáticos, os resultados podem apresentar viés significativo para pequenas amostras, conforme evidenciado nas simulações de Monte Carlo realizadas por Barnejee et alii (1993) e Inder (1993), citado por Parente e Carvalho (1999).

Portanto, na presença de quebra estrutural, teste de raiz unitária como Perron (1989) e Zivot e Andrews (1992), pode ser mais compatíveis do que os utilizados. E no caso do método de cointegração pode-se tentar usar o método proposto por Hansen e Johansen (1993) e a combinação de cointegração com processos markovianos formulada por Kolzig (1996) citado por Rodolfo (2008). 


\section{6 - REFERÊNCIAS BIBLIOGRÁFICAS}

CARVALHO, A., PARENTE, M. A. Estimação de Equações de Demanda de Importação por Categoria de Uso para o Brasil (1978/1996). Brasília, IPEA, Texto para Discussão n. 636, abril de 1999.

CUTHBERTSON, K.,HALL,S.G. e TAYLOR,M.P. (1992), Applied Econometric Tecnhiques,Havester Wheatsheaf, Londres. 1992

ENDERS, W. Applied econometric time series. Jonh Willey \& Sons, Inc., 1995.

ENGLE,R.F \& GRANGER, C.W.J.(1987).'Cointegration and Error Correction Representation Estimation, and Testing". Econometrica 55 (March 1987), 251-76

FERREIRA, A.H.B. Testes de Estabilidade para a Função Demanda de Importações. Revista Brasileira de Economia, 48(3): julho/setembro, 1994, p. 355-70.

GUJARATI, D. Basic Econometrics. 3 ed., McGraw-Hill, 1995.

HENDRY, D.F. Econometric modelling with cointegrating variables: an overview. Oxford Bulletin of Economics and Statistics, v. 48, n. 3, p. 201-212, 1986.

JOHANSEN, S. Statistical analysis of cointegrating vectors. Journal of Economic Dynamics and Control, v. 12, p. 231-254, 1988.

JOHANSEN, S., JUSELIUS, K. Maximum likelihood estimation and inference on

cointegration - with application to the demand for money. Oxford Bulletin of Economics and Statistics, v. 52, p. 169-210, 1990.

MACKINNON, J. G. Critical values for cointegrantion tests. In: ENGEL, R. F., e GRANGER,C. W. J. Long-run economic relantionships. Oxford University Press, 1991. p.267-276.
PERRON, P. The Great Crash, the Oil Price Shock, and the Unit Root Hypothesis.

Econometrica, v. 57, n. 6, novembro, 1989, p.

1361-1401.

PINDYCK, R., RUBINFELD., D. Econometric Models and Economic Forecast. 2 ed., McGrawHill, 1981.

PORTUGAL, M.S. Um Modelo de Correção de Erros para a Demanda por Importações

Brasileiras. Pesquisa e Planejamento Econômico, 22(3), dezembro, 1992.

RESENDE, M. F. C. Crescimento Econômico, Disponibilidade de Divisas e Importações Totais e por Categoria de Uso no Brasil: Um Modelo de Correção de Erros. Instituto de Pesquisa Econômica Aplicada, março de 2000

Zivot, E. \& D.W.K. Andrews.(1992). "Further Evidence on the Great Crash, the Oil Price Shock and the UnitRoot Hypothesis," Journal of Business and Economic Statistics 10, 251-270. 